\title{
Paraneoplastic Pemphigus: A Case Report
}

\author{
Shah N¹, Paudel R², Rijal A ${ }^{3}$ \\ ${ }^{1}$ Assistant Professor; 2Junior Resident; ${ }^{3}$ Professor, Department of Dermatology and Venerology, BP Koirala Institute of \\ Health Sciences, Dharan, Nepal
}

\begin{abstract}
Paraneoplastic pemphigus (PNP) is a rare autoimmune bullous disease associated with underlying neoplasms. Targetoid lesions, intractable stomatitis and refractory course of disease are some of the characteristic features of paraneoplastic pemphigus. It is usually associated with lymphoproliferative disorders, and rarely with solid tumors. We present a case of a 35 years old female with a six-months history of recurrent, severe, recalcitrant stomatitis and widespread blistering and erosions with involvement of palms and soles. A provisional diagnosis of PNP was made based on clinical features. Investigations for an underlying neoplasm revealed presence of solid lesion of size $6.7 \times 6.4 \mathrm{~cm}$ in left adnexa, likely an ovarian tumor. PNP should be considered in the differential diagnoses in severe atypical mucocutaneous manifestations of pemphigus vulgaris and diagnostic screening for search for an underlying tumor should be performed.
\end{abstract}

Key words: Acantholysis; autoantibodies; erythema multiforme; paraneoplastic pemphigus

\section{Introduction}

$\mathrm{P}$ araneoplastic pemphigus is a rare autoimmune bullous disease characterized by painful mucosal ulcerations and polymorphous skin lesions in association with an underlying neoplasm. It is most often associated with lymphoproliferative disorders. Association with solid malignancies is rare. Herein, we describe a case of paraneoplastic pemphigus associated with a solid ovarian tumor.

\section{Case report}

A 35 years old female presented with dusky macules, widespread erosions and vesicobullous lesions over body with oral, ocular and genital mucosal erosions for three weeks. She had severe, recalcitrant stomatitis for last six months. She had multiple fluid filled vesicles, bullae and erosions along with targetoid lesions on the trunk and extremities, predominantly upper back, neck and chest (Figure 1). She had erythematous macules and erosions on the palms and soles also. There were multiple erosions over the lips, buccal mucosa, palate, tongue, eyelids and genital mucosa (Figure 2). The lesions on the lips and eyelids were covered with yellowish slough and hemorrhagic crusts. Conjunctival congestion with yellowish discharge was also present.

Address of Correspondence:

Dr. Nidhi Shah

Department of Dermatology and Venereology.

BPKIHS

Ghopa Camp, Dharan, Sunsari, Nepal

E-mail: nidhishah.md@gmail.com
She had no known chronic disease or prior history of blistering lesions. She had no history of any drug intake. Baseline investigations were within normal limits. Initially provisional diagnoses of erythema multiforme and Stevens Johnsons syndrome was made. However, there was no history of any drug intake and any history of herpes labialis. In addition, HSV IgM was negative whereas HSV IgG was positive. Skin biopsy showed suprabasal clefting with floor lined by a single layer of basal cells. A diagnosis of paraneoplastic pemphigus (PNP) was made on clinical and histopathological features. Autoantibodies detection could not be done. Computed Tomography scan done for malignancy survey showed presence of a solid lesion of size 6.7 $X 6.4 \mathrm{~cm}$ in left adnexa likely a solid ovarian tumor. However, CA-125 was within normal limits. A diagnosis of PNP likely due to ovarian tumor, was made based on the details. The patient improved after 2 months

Submitted: $1^{\text {st }}$ June 2017

Accepted: $15^{\text {th }}$ August 2017

Published: $21^{\text {st }}$ March 2018

How to cite this article

Shah N, Paudel R, Rijal A. Paraneoplastic pemphigus: A case report. Nepal Journal of Dermatology Venereology and Leprology. 2018;16(1):57-8. doi: http://dx.doi.org/10.3126/ njdvl.v16i1.19412

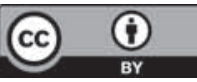

Licensed under CC BY 4.0 International License which permits use, distribution and reproduction in any medium, provided the original work is properly cited. 
of aggressive treatment with systemic corticosteroids including dexamethasone-cyclophosphamide pulse (DCP) therapy. She was counselled regarding prognosis of the disease and planned for surgical excision of the ovarian tumor following further improvement of the skin condition. Hence, she was discharged on oral corticosteroids and daily cyclophosphamide, and advised to follow in two weeks. She returned only after three weeks, with significant worsening of the skin lesions, and probable secondary infection. Her pulse and blood pressure were also low at this point, likely due to septic shock. She was advised for admission in the intensive care unit, but due to unavailability of beds in our hospital, could not be done. Patient's attendants were unwilling to take her elsewhere for the intensive care management, and chose to take her home against medical advice. Henceforth, the patient was lost to follow up.

\section{Disaussion}

In a majority of patients with PNP, the course is progressive and often fatal, even if the tumor is excised completely. A sizeable proportion of patients develop respiratory failure owing to damage to the bronchial epithelium due to acantholysis. ${ }^{1}$ The major associations of paraneoplastic pemphigus are lymphoproliferative disorders, however less commonly may be caused by other malignancies including soft tissue sarcomas, gastro-intestinal stromal tumors, pancreas, uterine and hepatocellular carcinomas. ${ }^{2,3}$ Association with a solid ovarian tumor was found in our case which was not characterized as the excision of the tumor could not be done. In our case, the patient had recalcitrant stomatitis, with erosions on the lips, and eyelids, and conjunctival involvement along with worsening of the skin lesions and the general condition of the patient at follow up. The worsening of the general condition may have been due to the malignancy itself.

\section{Condusion}

Paraneoplastic pemphigus should be considered in severe atypical mucocutaneous manifestations of

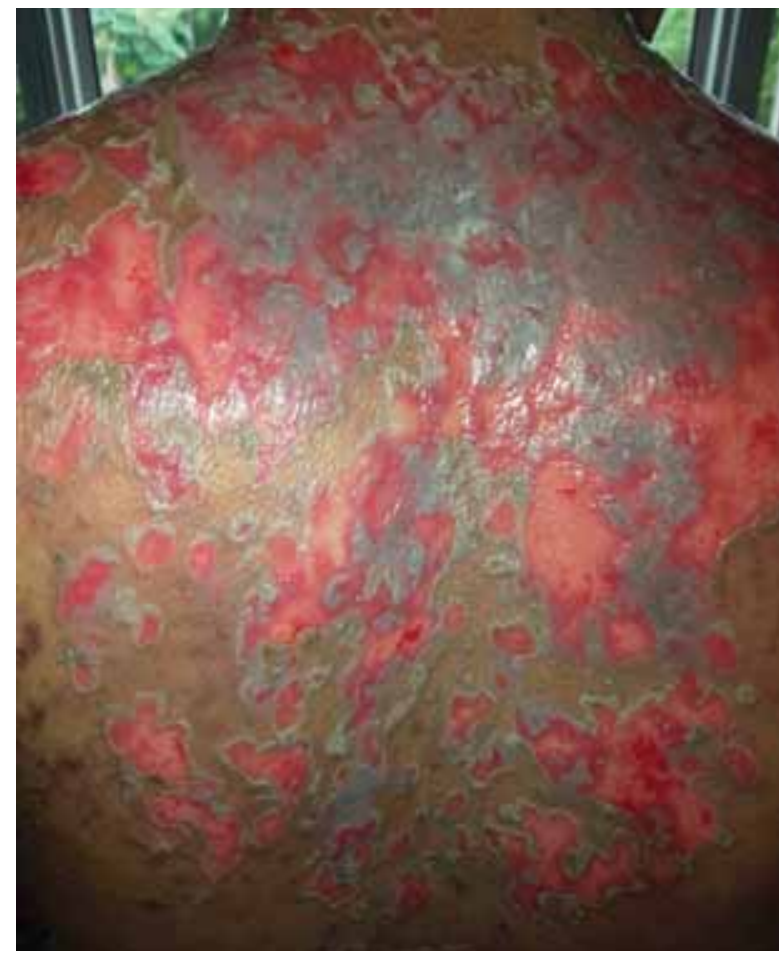

Figure 1: Erosions on the back with dusky erythematous macules on intact skin.

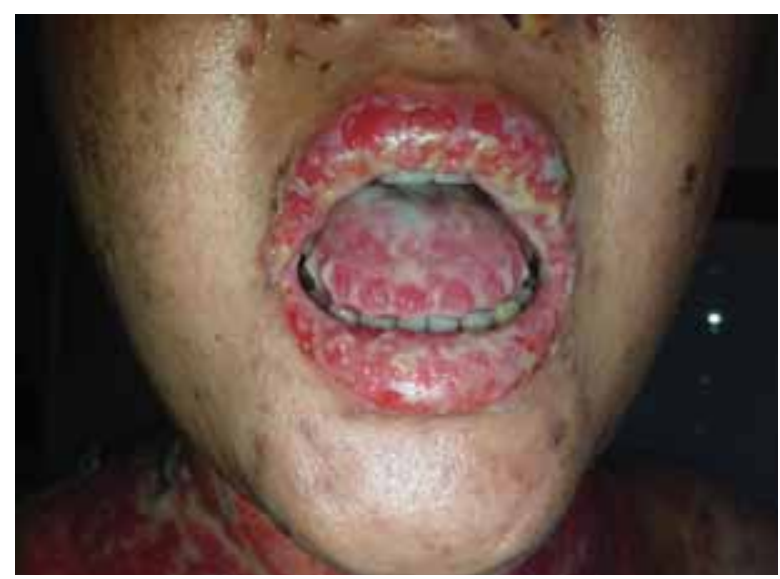

Figure 2: Severe stomatitis along with erosions on the tongue.

pemphigus vulgaris and diagnostic screening for search for an underlying tumor should be performed.

Financial disclosure: None.

Conflicts of interest to disclosure: None declared.

\section{References}

1. Tirado-Sánchez A, Bonifaz A. Paraneoplastic Pemphigus. A Life-Threatening Autoimmune Blistering Disease. Actas Dermosifiliogr. 2017;108(10):902-10. https://doi.org/10.1016/j. ad.2017.04.024

2. Kahawita IP, Fernando MS, Sirimanna GM, Fernando R, de Silva MV. Paraneoplastic

pemphigus associated with inflammatory myofibroblastic tumor. Int J Dermatol. 2006;45(11):1394-6. https://doi.org/10.1111/ j.1365-4632.2006.03109.x

3. Masu T, Okuyama R, Tsunoda T, Hashimoto T, Aiba S. Paraneoplastic pemphigus associated with malignant gastrointestinal stromal tumour. Acta Derm Venereol. 2010;90(1):89-90. https://doi. org/10.2340/00015555-0739 